\title{
3 Research Soure \\ Identifying Psychological Intervention Points for Alexithymia Based on the Process of Emotional Expression
}

\section{Toshiko Yasuda}

Kindai University

Hiromichi Matsuoka

National Cancer Center

Ryo Sakamoto

Kindai University

Atsuko Koyama ( $\nabla$ a-koyama@med.kindai.ac.jp )

Department of Psychosomatic Medicine, Kindai University, Faculty of Medicine https://orcid.org/00000003-3411-3313

\section{Research}

Keywords: alexithymia, psychosomatic disorder, the process of emotional expression

Posted Date: May 17th, 2021

DOI: https://doi.org/10.21203/rs.3.rs-513281/v1

License: (c) (i) This work is licensed under a Creative Commons Attribution 4.0 International License. Read Full License 


\section{Abstract}

Background: Alexithymia is a central concept in the field of psychosomatic disease. Patients afflicted with alexithymia have difficulties identifying feelings preventing them from responding well to psychotherapy. This study aims to evaluate in detail which steps of emotional expression (Steps 1-5) proposed by Kennedy-Moore et al. (Kennedy-Moore E and Watson JC. Expressing emotion. In: Salovey P, editor. Expressing Emotion. New York: Guilford; c1999. p. 8-18) are disrupted in alexithymia to enable the administration of effective treatment to such patients and identify appropriate methods of intervention for each step.

Methods: To investigate the relationship between the Japanese version of the Difficulties in Emotion Regulation Scale (J-DERS) total score and subscales and the 20 -item version of the Toronto Alexithymia Scale (TAS-20) subscales, multiple linear regression was performed using the former as dependent variables. The psychological examination records of eligible patients were retrospectively investigated. To evaluate the effect of alexithymia on each step of the process of emotional expression, the scores on the total and subscale J-DERS of the group that scored high on TAS-20 were compared with those of the group that scored low on TAS-20.

Results: Of the 188 total subjects, 106 (56\%) were included in the analysis. The median total J-DERS score was significantly different $(p<0.01)$ between the high-scoring group (defined as 52 points or higher) and the low-scoring group on the TAS-20, with a median score of 42.0 (interquartile range (IQR) 52.8 [upper limit]-31.0 [lower limit]) and 29.5 (IQR 37.3-23.0), respectively. Similarly, a significant difference was seen with each subscale $(p<0.01)$. Thus, disruption of the process of emotional expression in alexithymic patients is not only observed in Step 3 but also in Steps 4 and 5. Of the three TAS-20 subscales, only difficulty in identifying feelings correlated with the J-DERS total score and subscales $(p<0.01)$.

Conclusions: The results indicate that, when dealing with alexithymic patients individually in a clinical setting, therapeutic intervention should be adapted to Steps 3, 4, and 5 as appropriate for the patient, and that assessing each step using J-DERS may be more clinically useful.

\section{Background}

The construct of alexithymia includes the following features: 1) difficulty in identifying and describing feelings; 2) difficulty in distinguishing between feelings and the bodily sensations of emotional arousal; 3) constricted imaginative processes; and 4) an externally oriented cognitive style [1]. Sifneos et al. coined the term "alexithymia" as a central concept to describe such a psychosomatic disorder, which was present in patients with physical disease, developed and progressed in close correlation with psychosocial factors, and exhibited organic or functional disturbances [2, 3]. Alexithymia has been reported to correlate not only with psychosomatic disease but also with physical diseases such as diabetes mellitus and chronic pain [4,5]. Accordingly, therapeutic intervention for alexithymia patients is 
an important clinical topic. Since psychotherapies, such as cognitive-behavioral therapy or supportive psychotherapy, are administered based on the premise that the subjects are aware of their emotions or stress, it is difficult to apply these therapies to an appreciable effect with alexithymic patients [6-8]. Therefore, we believe that possible therapeutic interventions that focus on the processes leading up to the point at which emotions are expressed must be investigated. Kennedy-Moore and Watson described the process of emotional expression, in which emotional experiences brought about by emotion-eliciting stimuli are expressed, as comprising the following five steps:

1. STEP 1 [Initial prereflective reaction]

2. STEP 2 [Conscious perception of response]

3. STEP 3 [Labeling \& interpretation of response]

4. STEP 4 [Evaluation of response as acceptable]

5. STEP 5 [Perceived context for expression]

Kennedy-Moore and Watson proposed that alexithymia was a disruption of the third step: labeling \& interpretation of response (Fig. 1) [9]. Furthermore, if a disruption clinically existed in Step 3, additional disruptions may exist in a subsequent step or steps. This indicates that, when attempting to administer an effective therapeutic intervention in a case of psychosomatic or physical disease in which alexithymia is involved, it is necessary to closely investigate which steps are disrupted and to clearly identify what methods of intervention are appropriate for each step. The 20-item version of the Toronto Alexithymia Scale (TAS-20) is a self-administered scale developed by Bagby et al [10-13]. The TAS-20 is based on three factors: Difficulty Identifying Feelings (DIF), Difficulty Describing Feelings (DDF), and Externally Oriented Thinking (EOT) $[13,14]$, and uses the total scores to assess alexithymia. DIF seems to correspond to a disruption in Step 3 in Kennedy-Moore and Watson's process of emotional expression. However, a similar comparison cannot be made for Steps 4 or 5 in the process of emotional expression described above. In this respect, the Japanese version of the Difficulties in Emotion Regulation Scale (JDERS) (Table 1) [15] seems to better reflect the process of emotional expression (Fig. 2). Therefore, we believe that using the new J-DERS indicators could make it possible to evaluate how the process of emotional expression is affected in each step. Currently, no studies have used J-DERS to evaluate how each step of the process of emotional expression is affected in cases of alexithymia diagnosed using total TAS-20 scores. This study aimed to search for effective modes of therapeutic intervention for alexithymia, which is a condition closely connected with psychosomatic disease. For this purpose, we decided to use the existing TAS-20 and J-DERS scales to investigate which steps in the process of emotion expression were disrupted. 


\section{Q Lack of Emotional Awareness}

\section{1) I am clear about my feelings. (r)}

2) I have difficulty making sense of my feelings.

3) I know exactly how I am feeling. (r)

\section{4) I am confused about how I feel.}

$\otimes$ Nonacceptance of Emotional Responses

1) When I'm upset, I become angry with myself for feeling that way.

2) When I'm upset, I become embarrassed for feeling that way.

3) When I'm upset, I become irritated with myself for feeling that way.

4) When I'm upset, I start to feel very bad about myself.

\section{Q Limited Access to Emotion Regulation Strategies}

1) When I'm upset, I believe that I will remain that way for a long time.

2) When I'm upset, I believe that I'll end up feeling very depressed.

3) When I'm upset, I believe that there is nothing I can do to make myself feel better.

4) When I'm upset, it takes me a long time to feel better.

\Impulse Control Difficulties

1) When I'm upset, I have difficulty getting work done.

2) When I'm upset, I get out of control.

3) When I'm upset, I lose control over my behaviors.

4) When I'm upset, I have difficulty thinking about anything else.

Yamada \& Sugie. Jpn J Psychosom Med. 2013

J-DERs was used with its creators' permission. 


\section{Q Lack of Emotional Awareness}

\section{1) I am clear about my feelings. (r)}

\section{2) I have difficulty making sense of my feelings.}

3) I know exactly how I am feeling. (r)

\section{4) I am confused about how I feel.}

The J-DERS is a Japanese scale for evaluating potentially clinically problematic disruption in four abilities. Described by Gratz and Roemer, the scale reflects the process of emotional regulation from emotional arousal to emotional expression, using the following subscales:

(1) lack of emotional awareness, (2) nonacceptance of emotional responses, (3) limited access to emotion regulation strategies,

(4) impulse control difficulties.

Answers are given on a 5-point Likert scale ranging from 1 (almost never) to 5 (always)

Higher scores indicate greater difficulty regulating emotions

(r) Indicates a reverse-score item.

The DERS contains six subscales:

1. Nonacceptance of Emotional Response (NONACCEPTANCE)

2. Difficulties in Engaging in Goal-Directed Behavior (GOALS)

3. Impulse Control Difficulties (IMPULSE)

4. Lack of Emotional Awareness (AWARENESS)

5. Limited Access to Emotion Regulation Strategies (STRATEGIES)

6. Lack of Emotional Clarity (CLARITY)

Gratz \& Roemer. J Psychopathol Behav Assess. 2004

\section{Methods}

\section{Subjects}

First-time patients who presented to Kindai University Hospital Department of Psychosomatic Medicine Outpatient Clinic between February 2018 and June 2019, did not have dementia or other mental illnesses, and met the criteria below were included in the study. The eligibility criteria included an age of 16 years or higher and no refusal to provide information. The study plan was announced prior to the commencement of the project on the website of the Department of Psychosomatic Medicine, Kindai University Faculty of Medicine (https://www.med.kindai.ac.jp/ryonai/) to provide an opportunity for those not wishing to participate to express their refusal. The exclusion criteria included refusal to participate or a failure to provide written consent despite agreeing to participate. Exclusion diagnosis included i) Neurocognitive 
Disorders, ii) Depressive Disorders, iii) Obsessive-Compulsive and Related Disorders, iv) Neurocognitive Disorders, v) Feeding and Eating Disorders, vi) Personality Disorders, and vii) Anxiety Disorders. Diagnosis was using Diagnostic and Statistical Manual of Mental Disorders, Fifth Edition (DSM-5).

\section{Psychological parameters}

\section{0-item version of The Toronto Alexithymia Scale, TAS-20}

To assess alexithymia in this study, we used the TAS-20, which is a self-administered scale developed by Bagby et al [10-13]. The TAS-20 is based on three factors, namely, DIF, DDF, and EOT $[13,14]$. The TAS20 's reliability and validity have been previously verified internationally. The test comprises 20 statements that are graded from one (strongly disagree) to five (strongly agree). The total scores are used to assess alexithymia, and a sum of greater than or equal to 61 points indicates alexithymia, between 52 and 60 indicates an intermediate zone, while less than or equal to 51 points or below indicates non-alexithymia. In this study, patients with a TAS-20 score of 52 points or higher (which covers both the alexithymia and intermediate zones) were grouped in the TAS-20 high-scoring group, and patients with a score of 51 or lower were grouped in the TAS-20 low-scoring group.

\section{The Difficulties in Emotion Regulation Scale of Japanese version, J-DERS}

As shown in Table 1, to assess difficulties in emotion regulation in this study, the J-DERS, which is a Japanese version of the DERS, was used [15]. The J-DERS comprises four subscales (awareness, clarity, goals, and impulse) corresponding to the same subscales in the six-scale DERS [16]. The subscales measure a patient's ability to regulate emotion in four areas, comprehensively covering the process of emotion regulation from arousal to expression [16]. The manner in which the J-DERS subscales for (1) lack of emotional awareness, (2) nonacceptance of emotional responses, (3) limited access to emotion regulation strategies, and (4) impulse control difficulties are thought to correspond to the process of emotional expression outlined by Kennedy-Moore et al. is shown in Fig. 2. Participants were asked to indicate on a five-point Likert-type scale depending on how the items applied to them, with $1=$ almost never, 2 = sometimes, $3=$ about half the time, $4=$ most of the time, and $5=$ almost always. Higher scores indicate greater difficulties in emotion regulation.

\section{Procedures and statistics}

Psychological assessments using TAS-20 and J-DERS were administered with the medical questionnaire that patients filled in before being examined at their initial visit to our hospital. We then retrospectively investigated this data based on our medical records for patients who met the eligibility criteria. To evaluate how the presence of alexithymia affected each step of the process of emotional expression, the comparison between the mean J-DERS total scores and subscale scores of the group that scored highly on the TAS-20 (TAS-20 total scores of $\geq 52$ ) and those of the group that scored low on the TAS-20 (TAS20 total scores of $\leq 51$ ) was set as the primary outcome. The Mann-Whitney $U$ test was used and a pvalue less than 0.01 was considered to be statistically significant. For the secondary outcome, we 
investigated factors correlated with the J-DERS total score and subscales. Forced-entry multiple linear regression was performed using the J-DERS total scores and subscales as dependent variables and age, sex, years of education, and TAS-20 subscale scores as independent variables. SPSS V.25 (SPSS, Inc., Chicago, IL, USA) was used to statistically analyze all data. This study was conducted in accordance with the principles of the Declaration of Helsinki and Japan's ethical guidelines for clinical research and approved by the Clinical Research Preliminary Review Board of the Kindai University Ethics Committee (Approval No. R2-073).

\section{Results}

Figure 3 shows how the subjects were selected. Out of 188 initial candidates, 152 met the eligibility criteria. Out of these, 46 met the exclusion criteria; thus, only 106 cases (56\%) were finally included in the analysis. The patient backgrounds are shown in Table 2. Patient ages ranged from 16 to 88 years with a mean age of 50.5. The most common main complaint was pain, which was reported by 40 patients (38\%), followed by digestive symptoms (diarrhea, abdominal pain, or heartburn accompanying enterokinesis) in 19 patients (18\%), dizziness in 13 (12\%), and numbness in $13(12 \%)$. The mean TAS-20 and J-DERS total and subscale scores and their standard deviations are shown in Table 3. J-DERS scores in the TAS-20 high and low groups are shown in Table 4 . There were 60 subjects (57\%) in the group that scored a total of $\geq 52$ on the TAS-20 (assigned as the TAS-20 high group), and 46 subjects (43\%) in the group that scored a total of $\leq 51$ (assigned as the TAS-20 low group). A significant difference $(p<0.01)$ was found in the median total J-DERS scores between the TAS-20 high and low groups, which was 42.0 (IQR 52.8-31.0) and 29.5 (37.3-23.0), respectively. For the TAS-20 high and low groups, the median subscale score for lack of emotional awareness, nonacceptance of emotional responses, limited access to emotion regulation strategies, and impulse control difficulties was $11.0(13.0-8.3)$ and 7.0 (9.0-6.0), $11.0(15.0-8.0)$ and $7.0(10.0-4.0), 10.0(14.0-8.0)$ and $8.0(10.0-6.0)$, and 11.0 (13.0-8.0) and 7.0 (10.0-5.0), respectively. A significant difference was observed between the two groups for each of the subscales $(p<0.01)$. The factors related to J-DERS total and subscale scores and the results of a multivariate analysis applied to the covariates age, sex, years of education, and TAS-20 subscales are shown in Additional file 1. The normality of the variables was verified in advance using the Shapiro-Wilk test, yielding a p-value of 0.283 , which is not a significant deviation from a normal distribution. There was no multicollinearity; none of the variance inflation factors exceeded 10. A correlation matrix was plotted, but none of the variables showed a correlation of over $|r|>0.9$. Since ANOVA returned significant results, and the Durbin-Watson statistic returned values near 2 for each of the variables, the probability of randomness was high, and none of the predicted values fell outside \pm 3 standard deviations of the measured values. As summarized in Additional file 1, out of the three TAS-20 subscales (DIF, EOT, and DDF), only DIF was correlated with the J-DERS total score and each of the J-DERS subscales (lack of emotional awareness, nonacceptance of emotional responses, limited access to emotion regulation strategies, and impulse control difficulties) $(p<0.01)$. Conversely, EOT and DDF had no correlation with any of the J-DERS subscales. 
Table 2

Clinical characteristics of the participants

Number of participants $(n=$ 106)

Age (years)

Mean (range)

$50.5(16-88)$

Education (years)

Mean (range)

$13.0(9-16)$

Sex, n (\%)

Men

Women

$71(67)$

Symptoms, n (\%)

Pain

Digestive symptoms

(diarrhea, abdominal pain, or heartburn accompanying enterokinesis)

Dizziness

$13(12)$

Numbness

$13(12)$

Palpitations

$11(10)$

Others

$10(9)$ 
Table 3

TAS-20 and J-DERS total scores and subscales of the participants $(n=106)$

\begin{tabular}{|lll|}
\hline & Mean & Standard Deviation \\
\hline TAS-20 & & \\
\hline TAS-20 Total & 53.3 & 9.8 \\
\hline Difficulty Identifying Feeling & 17.3 & 6.2 \\
\hline Difficulty Describing Feeling & 14.3 & 3.6 \\
\hline Externally Oriented Thinking & 21.7 & 3.3 \\
\hline J-DERS & & \\
\hline J-DERS Total & 37.9 & 13.3 \\
\hline Lack of Emotional Awareness & 9.3 & 3.3 \\
\hline Nonacceptance of Emotional Responses & 9.6 & 4.2 \\
\hline Limited Access to Emotion Regulation Strategies & 9.7 & 4.0 \\
\hline Impulse Control Difficulties & 9.6 & 4.0 \\
\hline
\end{tabular}

Table 4

J-DERS total and subscale scores in the TAS-20 high/low groups (Mann-Whitney U test)

\begin{tabular}{|c|c|c|c|}
\hline & TAS-20 High Group & TAS-20 Low Group & \\
\hline Score & $\begin{array}{l}\text { Median (upper quartile I } \\
\text { lower quartile) }\end{array}$ & $\begin{array}{l}\text { Median (upper quartile I } \\
\text { lower quartile) }\end{array}$ & \\
\hline J-DERS Total & $42.0(52.831 .0)$ & $29.5(37.323 .0)$ & $\begin{array}{l}\mathrm{p}< \\
0.01\end{array}$ \\
\hline Lack of Emotional Awareness & $11.0(13.08 .3)$ & $7.0(9.06 .0)$ & $\begin{array}{l}\mathrm{p}< \\
0.01\end{array}$ \\
\hline $\begin{array}{l}\text { Non-acceptance of Emotional } \\
\text { Responses }\end{array}$ & $11.0(15.08 .0)$ & $7.0(10.04 .0)$ & $\begin{array}{l}p< \\
0.01\end{array}$ \\
\hline $\begin{array}{l}\text { Limited Access to Emotion } \\
\text { Regulation Strategies }\end{array}$ & $10.0(14.08 .0)$ & $8.0(10.06 .0)$ & $\begin{array}{l}\mathrm{p}< \\
0.01\end{array}$ \\
\hline Impulse Control Difficulties & $11.0(13.08 .0)$ & $7.0(10.05 .0)$ & $\begin{array}{l}\mathrm{p}< \\
0.01\end{array}$ \\
\hline
\end{tabular}

\section{Discussion}


To our knowledge, this study is the first to have used J-DERS to evaluate the patient's function at each step of the process of emotional expression that is also affected by the presence or absence of alexithymia as assessed by TAS-20. Initially, upon comparison of the TAS-20 high- and low- scoring groups, we expected to only find a disruption in the third step of the process of emotional expression described by Kennedy-Moore and Watson (Fig. 1) [9]. In other words, we expected to only find a difference in the scores relating to the J-DERS subscale for lack of emotional awareness when comparing the two groups. However, we found significant differences both in the total J-DERS score and also for each of its subscales. This is the most important point to consider in this study, as it suggests that when there is a disruption of Step 3 (labeling and interpretation of response) illustrated in Fig. 2, functioning in the following steps can also be affected, which may also lead to disruption of Steps 4 and 5. Gratz and Roemer proposed that emotion regulation involved the following four abilities: (a) awareness and understanding of emotions, (b) acceptance of emotions, (c) ability to control impulsive behaviors and behave in accordance with desired goals when experiencing negative emotions, (d) ability to use situationally appropriate emotion regulation strategies flexibly to modulate emotional responses as desired in order to meet individual goals and situational demands [15]. These correspond to the four subscales of the J-DERs outlined in Table 1: (1) lack of emotional awareness, (2) nonacceptance of emotional responses, (3) limited access to emotion regulation strategies, and (4) impulse control difficulties [16]. The results of the present study suggest that, in patients indicated to have alexithymia or be in the intermediate zone, emotion regulation is hindered with regard to each of these four abilities. This means that when dealing with individual alexithymic patients in the clinical setting, therapeutic interventions for the alexithymia should probably be adapted to Steps 3, 4, or 5 as appropriate for each patient. The TAS-20 has three subscales, namely, DIF, DDF, and EOT. Together with the process of emotional expression, these three concepts are fundamental to the concept of alexithymia. DIF seems to correspond to a disruption in Step 3, while DDF to a disruption in Step 4. We hypothesized that when alexithymic individuals fail to use emotion as an indicator to deal with events due to their inability to identify emotions, they tend to focus on the circumstances and details surrounding events [17] and fall into EOT. In this regard, we hypothesized that EOT also corresponds to a disruption in Step 3 (Fig. 4). A comparison of the TAS-20 and J-DERS subscales showed that although DIF was correlated with all of the J-DERS total and subscale scores, DDF and EOT were not. This essentially means that the TAS-20 subscales do not measure the individual contributions of Steps 3, 4, and 5. In other words, TAS-20 essentially describes alexithymia as the sum of effects in Steps 3, 4, and 5. In accordance with this, our results indicate that using the J-DERS to evaluate each individual step may be more useful in the clinical setting. Since psychotherapies such as cognitive-behavioral therapy and supportive psychotherapy do not tend to produce good results with alexithymia, and based on the results of the present study, we suggest that alexithymia patients with a disruption in Step 3 could be prompted not to identify as "feelings" the "conscious perceptions of response" which occurs when emotions are aroused, but instead to notice the "bodily sensations" that they experience, and then to recognize, identify, and label these accordingly. For example, a potential method may be to prompt an alexithymia patient to write a description of an event, and then encourage them to learn what emotions would correspond to it. For a disruption in Step 4, a potential therapeutic intervention could involve using mindfulness to encourage 
the acceptance of emotion [18], while for a disruption in Step 5, a cognitive-behavioral treatment such as that proposed by Barlow [19] may be appropriate. In this way, therapeutic interventions can be tailored to target the disruptions in the various steps. We believe that if such therapies are successful, then alexithymic individuals may better their ability to express emotions, which can lead to allowing them to compare their emotions and consider how best to act, leading to a change in their behavior, and ultimately enabling them to better deal with potentially stressful situations and interactions. Administering therapeutic interventions tailored for the various steps in which disruptions exist when treating alexithymia and intermediate zone patients is important; however, further studies are required to investigate the emotional processing and modes of these therapeutic interventions. Moreover, it may be necessary to develop a new scale to evaluate the disruption of the process of emotional expression on an individual level. This study aimed to verify in which steps of the process of emotional expression disruptions existed in alexithymia-a condition closely connected with psychosomatic disorders and physical disease-and to clarify what methods would be appropriate for treating the disruptions in the various steps, so that effective therapeutic interventions can be administered. The results showed that in alexithymia, disruption can occur not only in Step 3, which is essentially the definition of the condition, but also in Steps 4 and 5. The present study had the following four limitations: First, this study assessed alexithymia using self-administered tests. Next, the presence of alexithymia was defined as a TAS-20 score of 52 or greater, which meant that the intermediate zone was also included in the TAS- 20 high group. Additionally, patients with mental disease that presented with mainly physical symptoms were may have been inadvertently included. Finally, this was a retrospective study of cases at a single institution, which means that questions could be raised with regard to its external validity and generalizability. However, as differences were observed between the TAS-20 high group (which encompassed both alexithymia and the intermediate zone) and the TAS-20 low group with respect to their total and subscale J-DERS scores, we can say that the present study did succeed in clarifying the fact that in alexithymia, disruption also occurs in Steps 4 and 5, and not only in Step 3 as was previously reported. The present study provides a useful starting point for developing therapeutic interventions for alexithymia.

\section{Conclusions}

We used J-DERS to investigate whether disruptions existed in various steps of the process of emotional expression in alexithymia, which is closely connected with psychosomatic disorders and physical disease, so that effective therapeutic interventions can be administered. The results of our study revealed that disruption occurs, not only in Step 3, which is essentially what defines alexithymia, but also in Steps 4 and 5 . The results show that it is important to implement various modes of therapeutic intervention as appropriate for the various steps. Although our results suggest that evaluating individual steps using JDERS is more useful in clinical settings, we recommend that future studies develop a new scale to provide more detailed assessments of disruption in the process of emotional expression in individual patients. 


\section{Abbreviations}

TAS-20: 20-item version of The Toronto Alexithymia Scale

DIF: Difficulty Identifying Feeling

DDF: Difficulty Describing Feeling

EOT: Externally Oriented Thinking

J-DERS: The Difficulties in Emotion Regulation Scale of Japanese version

DSM-5: Diagnostic and Statistical Manual of Mental Disorders, Fifth Edition

\section{Declarations}

\section{Ethics approval and consent to participate}

This study received the approval of the Clinical Research Preliminary Review Board of the Kindai University Ethics Committee (Approval No. R2-073). Although there were no opportunities to inform the research subjects that did not visit Kindai University Hospital during the period in which the study was conducted, we did inform and obtain the verbal consent of each research subject that visited during the study period when such an opportunity arose. All study procedures were carried out in accordance with the principles in the Declaration of Helsinki and its later amendments.

\section{Consent for publication}

Not applicable.

\section{Availability of data and materials}

Data sharing not applicable to this article as no datasets were generated or analyzed during the current study.

\section{Competing interests}

The authors declare that they have no competing interests.

\section{Funding}

The research did not receive any specific grants from funding agencies in the public, commercial, or notfor-profit sections.

\section{Authors' contributions}


TY and AK conceived the study and participated in its design. TY performed the statistical analysis. AK, $\mathrm{HM}$, and RS analyzed the data and helped draft the manuscript. All authors read and approved the final manuscript.

\section{Acknowledgements}

The authors thank Crimson Interactive Pvt. Ltd. (Ulatus) - www.ulatus.jp for their assistance in manuscript translation and editing.

\section{References}

1. Taylor GJ, Bagby RM, Parker JD. The alexithymia construct. A potential paradigm for psychosomatic medicine. Psychosomatics. 1991;32:153-64.

2. Japan Psychosomatic Medicine Education and Training Committee. New diagnostic guidelines for psychosomatic medicine. J Psychosom Med. 1991;31:541.

3. Sifneos PE. The prevalence of alexithymic characteristics in psychosomatic patients. Psychother Psychosom. 1973;22:255-62.

4. Shibata M, Ninomiya T, Jensen MP, Anno K, Yonemoto K, Makino S, et al. Alexithymia is associated with greater risk of chronic pain and negative affect and with lower life satisfaction in a general population: the Hisayama study. PLoS One. 2014;9:e90984

5. Chatzi L, Bitsios P, Solidaki E, Christou I, Kyrlaki E, Sfakianaki M, et al. Type 1 diabetes is associated with alexithymia in nondepressed, non-mentally ill diabetic patients: A case-control study. J Psychosom Res. 2009;67:307-13.

6. Pinna F, Manchia M, Paribello P, Carpiniello B. The impact of alexithymia on treatment response in psychiatric disorders: A systematic review. Front Psychiatry. 2020;16:1-21.

7. Blaettler LT, Stewart JA, Gubler DA, Egloff N, von Känel R, Holtforth M. Alexithymia moderates effects of psychotherapeutic treatment expectations on depression outcome in interdisciplinary chronic pain treatment. J Psychosom Res. 2019;122:69-72.

8. Rutten EA, Bachrach N, van Balkom AJ, Braeken J, Ouwens MA, Bekker MHJ. Anxiety, depression and autonomy-connectedness: The mediating role of alexithymia and assertiveness. Psychol Psychother. 2016;89:385-401.

9. Kennedy-Moore E, Watson JC. Expressing emotion. In: Salovey P, editor. Expressing Emotion. New York: Guilford; 1999. p. 8-18.

10. Bagby RM, Parker JD, Taylor GJ. The twenty-item Toronto Alexithymia Scale- I. Item selection and cross-validation of the factor structure. J Psychosom Res. 1994;38:23-32.

11. Bagby RM, Taylor GJ, Parker JD. The twenty-item Toronto Alexithymia Scale-ll. Convergent, discriminant, and concurrent validity. J Psychosom Res. 1994;38:33-40.

12. Bagby RM, Taylor GJ, Quilty LC, Parker JDA. Reexamining the factor structure of the 20 -item Toronto alexithymia scale: commentary on Gignac, Palmer, and Stough. J Pers Assess. 2007;89:258-64. 
13. Taylor GJ, Bagby RM, Parker JD. The 20-item Toronto Alexithymia Scale. IV. Reliability and factorial validity in different languages and cultures. J Psychosom Res. 2003;55:277-83.

14. Komaki G, Maeda M, Arimura T, Nakata A, Shinoda H, Ogata I, et al. The reliability and factorial validity of the Japanese version of the 20 -item Toronto alexithymia Scale (TAS-20). Jpn J Psychosom Med. 2003;43:839-46.

15. Gratz KL, Roemer L. Multidimensional assessment of emotion regulation and dysregulation: development, factor structure, and initial validation of the difficulties in emotion regulation scale. J Psychopathol Behav Assess. 2004;26:41-54.

16. Yamada K, Sugie M. Development and validation of Japanese version of difficulties in emotion regulation scale (J-DERS). Jpn J Psychosom Med. 2013;20:86-95.

17. Arimura T, Tamagawa K, Nozaki T, Komasaki G, Nishikata H, Takii M, et al. Development of the Structured Interview by the Modified Edition of Beth Israel Hospital Psychosomatic Questionnaire (SIBIQ) in Japanese edition to evaluate alexithymia. Jpn J Psychosom Med. 2002;42:259-69.

18. Lindsay EK, Creswell JD. Mindfulness, acceptance, and emotion regulation: Perspectives from Monitor and Acceptance Theory (MAT). Curr Opin Psychol. 2019;28:120-5.

19. Barlow DH, Allen LB, Choate ML. Toward a unified treatment for emotional disorders. Behav Ther. 2004;35:205-30.

\section{Figures}
Step 1
Step 2
Step 3
Step 4
Step 5

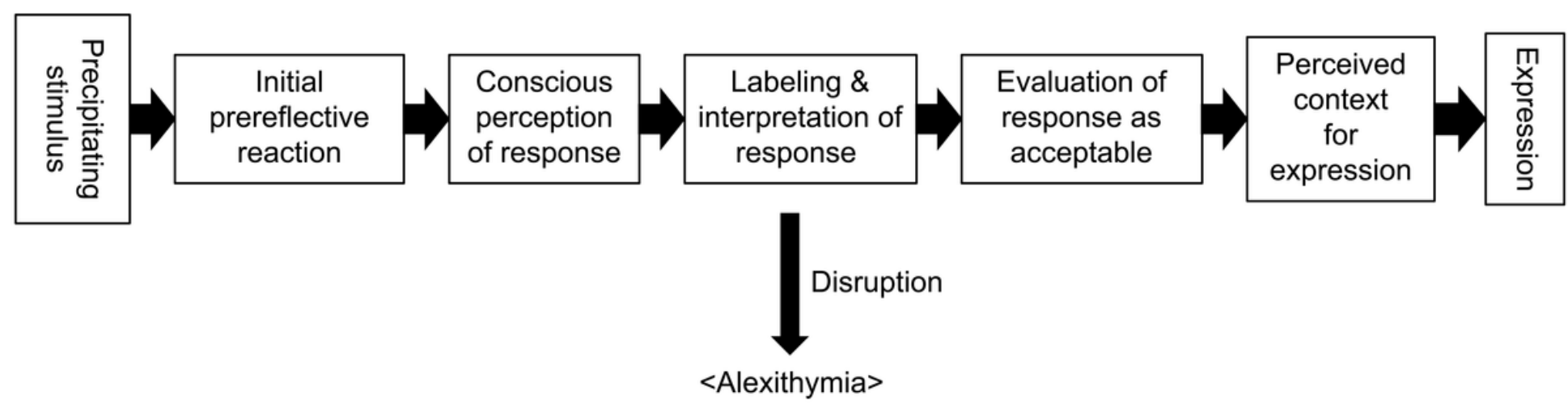

\section{Figure 1}

The process of emotional expression Five steps exist between affective arousal and emotional expression. STEP 1: Initial prereflective reaction When the emotion-eliciting stimulus is received, a reaction happens in which the stimulus is perceived, preconscious cognitive and emotional processing takes place, and emotional arousal occurs. This arousal is a bodily signal. STEP 2: Conscious perception of the response The individual observes the affective reaction caused by the bodily signal, and consciously recognizes this experience. Specific bodily signs such as a racing heart or shaking hands may be noticed. STEP 3: Labeling and interpreting the response If the affective response can be 
consciously perceived, then the experience undergoes cognitive processing and is labeled as an emotional experience, and an attempt is made to label and interpret it. Alexithymia is a disruption of this step. STEP 4: Evaluation of the response as acceptable If the affective response can be labeled and interpreted, then it is compared with the individual's beliefs and goals, and the individual decides whether or not to accept the feeling. STEP 5: Perceived context for expression If the individual perceives that revealing their feelings is possible or desirable in their interpersonal environment, they then ultimately express these feelings.

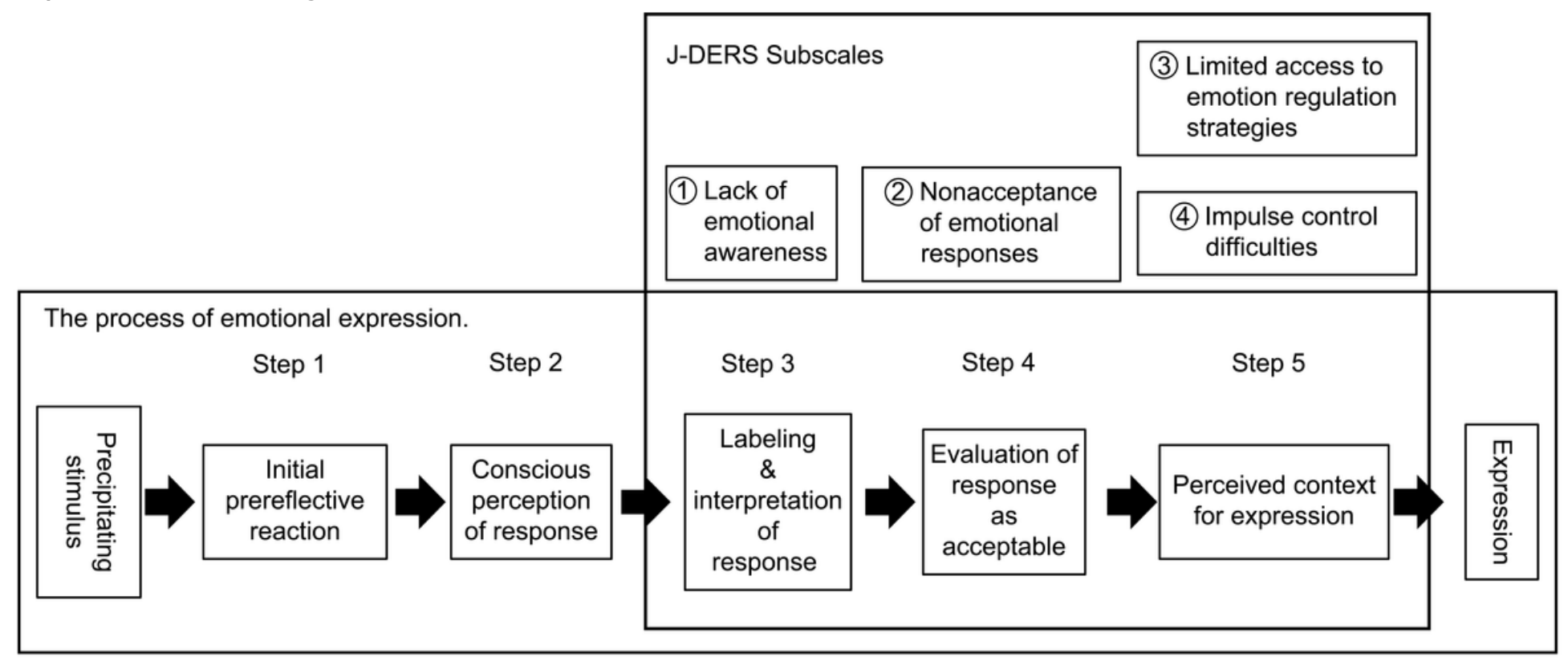

Figure 2

J-DERS and its relation to process of emotional expression It is thought that disruption in STEP 3 of the process of emotional expression equates to the J-DERS subscale 1. Lack of emotional awareness, disruption in STEP 4 equates to the J-DERS subscale; 2. Nonacceptance of emotional awareness, and disruption in STEP 5 equates to the J-DERS subscales; 3 . Limited access to emotion regulation strategies; and 4. Impulse control difficulties. 
First-time patients during study period (188)

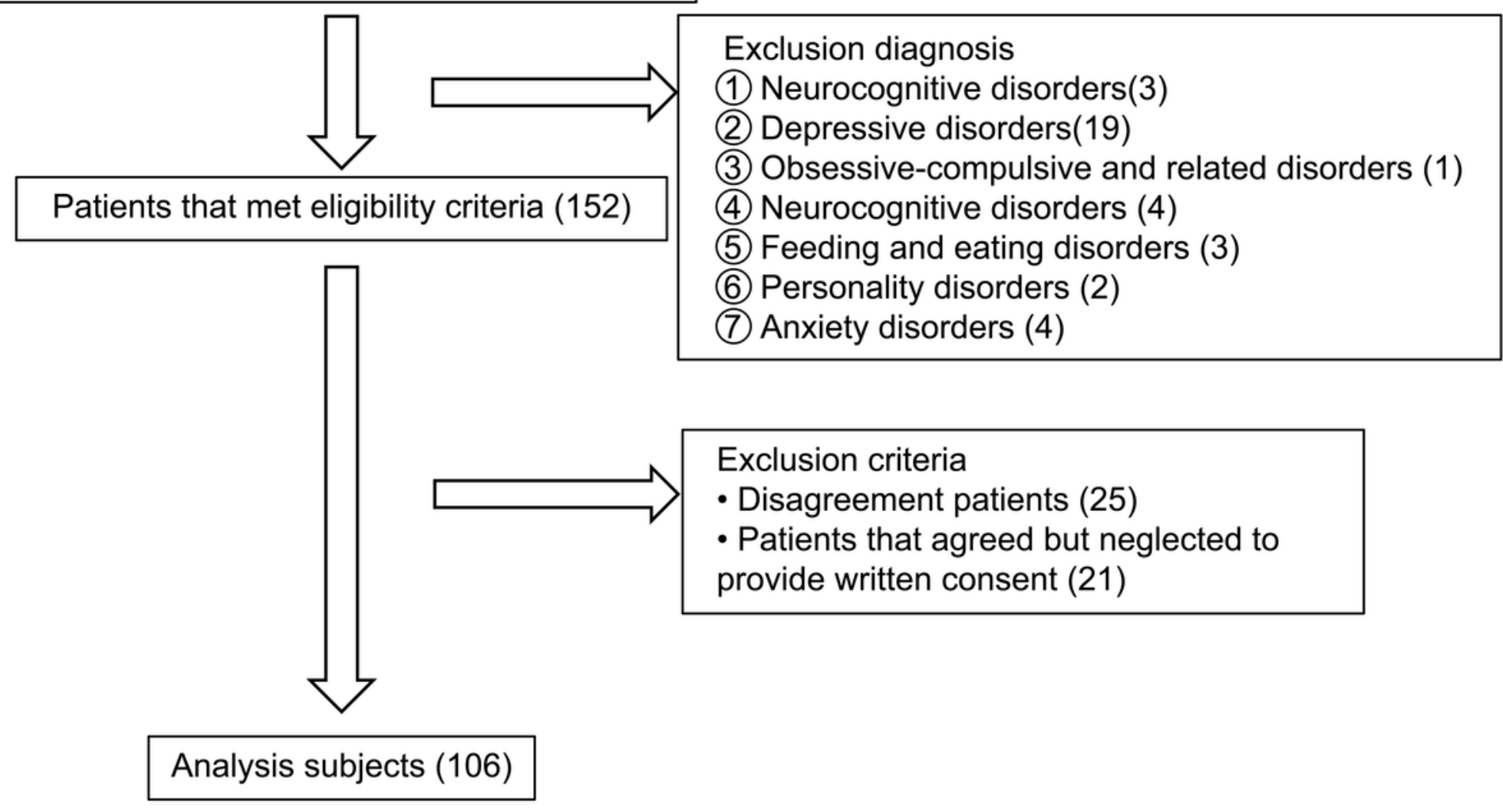

\section{Figure 3}

Flowchart of the study The subjects of the study were first-time patients referred to the Kindai University Hospital Department of Psychosomatic Medicine Outpatient Clinic in the period from February 2018 to June 2019 that did not have dementia or mental illness and that met the criteria below: Eligibility criteria: $\nabla 16$ years and over $\nabla$ No refusal to provide information Exclusion diagnosis: $\nabla$ Neurocognitive Disorders $\nabla$

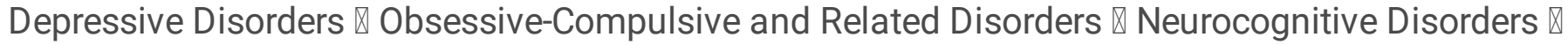
Feeding and Eating Disorders $₫$ Personality Disorders $₫$ Anxiety Disorders (DSM-5) Exclusion criteria: Disagreement patients, Patients who agreed but neglected to provide written consent 


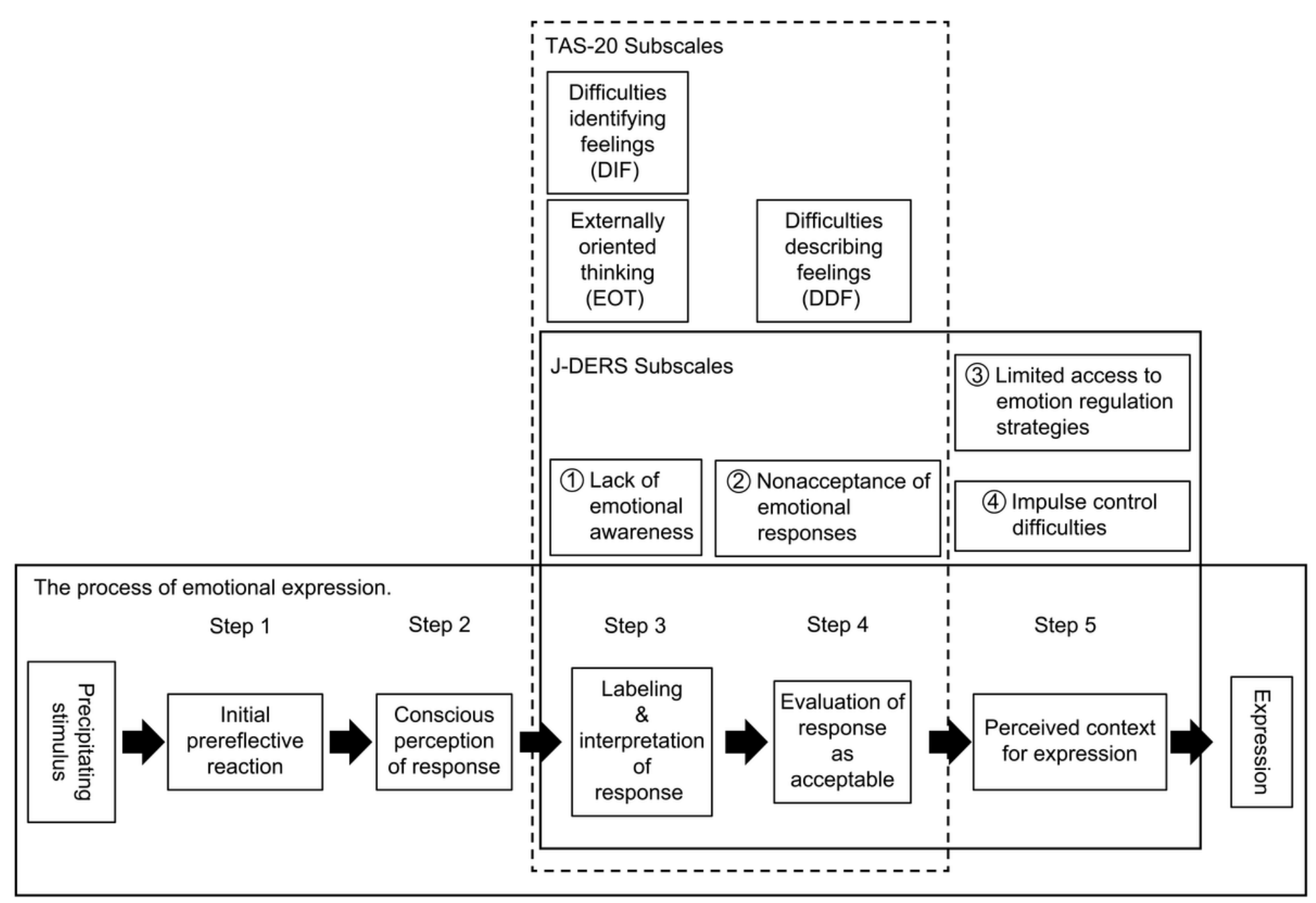

Figure 4

Flow for therapeutic interventions for alexithymia that focus on the process of emotional expression It is thought that disruption in STEP 3 of the process of emotional expression equates to the TAS-20 subscales Difficulties Identifying Feelings (DIF) and Externally Oriented Thinking (EOT), and disruption in STEP 4 equates to the TAS-20 subscale Difficulties Describing Feelings (DDF).

\section{Supplementary Files}

This is a list of supplementary files associated with this preprint. Click to download.

- Additionalfile1.xlsx 